\title{
Cuidado em saúde mental: uma reflexão da assistência às pessoas em sofrimento
}

\section{psíquico na atualidade}

\author{
Care in mental health: a reflection of assistance to people in psychic suffering currently
}

Atención en salud mental: un reflexión de la ayuda a personas en sufrimiento psíquico actualmente

Recebido: 03/10/2021 | Revisado: 13/10/2021 | Aceito: 22/10/2021 | Publicado: 24/10/2021

Elisângela Lopes de Faria

ORCID: https://orcid.org/0000-0001-8826-1675

Universidade Federal de São João del-Rei, Brasil

E-mail: elisangelalfaria@aluno.ufsj.edu.br

Joice Cristina de Paula

ORCID: https://orcid.org/0000-0002-3099-4136

Universidade Federal de São João del-Rei, Brasil

E-mail: joicedipaula@aluno.ufsj.edu.br

Juliana Almeida Menezes Moreira

ORCID: https://orcid.org/0000-0002-2384-9328

Universidade Federal de São João del-Rei, Brasil

E-mail: julianamenezesufsj@gmail.com

Lucas Alves Gontijo

ORCID: https://orcid.org/0000-0002-6005-8154

Universidade Federal de São João del-Rei, Brasil

E-mail: enf.lucasgontijo@aluno.ufsj.edu.br

Tamires Caroline Silva

ORCID: https://orcid.org/0000-0002-2980-8973

Universidade Federal de São João del-Rei, Brasil

E-mail: ta.csilva@ aluno.ufsj.edu.br

Edilene Aparecida Araújo da Silveira

ORCID: https://orcid.org/0000-0001-7378-2240

Universidade Federal de São João del-Rei, Brasil

E-mail: edileneap@ufsj.edu.br

\begin{abstract}
Resumo
A Reforma Psiquiátrica foi um processo histórico que teve como objetivo principal garantir a humanização do cuidado às pessoas que experimentam sofrimento psíquico. Este artigo faz uma busca na literatura nacional realizando uma reflexão sobre as vicissitudes da dimensão relacional do cuidado, a constituição da RAPS como instrumento de promoção de humanização em saúde, inserção social e rompimento de todo o estigma que envolve a saúde mental. O presente estudo tem como objetivo realizar uma reflexão sobre o cuidado em saúde mental proporcionado pela Rede de Atenção Psicossocial à Saúde (RAPS). Trata-se de um ensaio teórico que discute o cuidado em saúde mental na perspectiva da Reforma psiquiátrica tendo como referencial teórico o cuidado ontológico, visto sob a fenomenologia segundo Heidegger. A discussão acerca da temática, ocorre em dois momentos, refletindo sobre o papel cuidado e da Reforma Psiquiátrica na articulação de serviços de saúde mental. De forma que o cuidado envolve uma série de princípios teóricos e práticos que são necessários para o oferecimento de uma assistência mais humana à saúde. Mesmo diante dos retrocessos e fragilidades enfrentados pela Saúde Mental Brasileira é preciso que os profissionais reflitam e ressignifiquem a humanização do cuidado no âmbito da saúde mental. Os profissionais de saúde mental devem sempre lutar por uma assistência mais pautada na valorização do indivíduo, levando em consideração o contexto social, econômico e cultural do usuário.
\end{abstract}

Palavras-chave: Saúde mental; Serviços de saúde mental; Sistema Único de Saúde.

\begin{abstract}
The Psychiatric Reform was a historical process whose main objective was to ensure the humanization of care for people who experience psychological distress. This article searches the national literature, reflecting on the vicissitudes of the relational dimension of care, the constitution of RAPS as an instrument for promoting humanization in health, social inclusion and breaking all the stigma surrounding mental health. This study aims to reflect on the mental health care provided by the Psychosocial Health Care Network (RAPS). This is a theoretical essay that discusses mental health care from the perspective of the Psychiatric Reform, having as theoretical reference the ontological care, seen under the phenomenology according to Heidegger. The discussion about the theme occurs in two moments, reflecting on the role of care and the Psychiatric Reform in the articulation of mental health services. So, care involves a series of theoretical and practical principles that are necessary to offer a more humane assistance to
\end{abstract}


health. Even in the face of setbacks and weaknesses faced by Brazilian Mental Health, professionals need to reflect and reframe the humanization of care in the context of mental health. Mental health professionals should always strive for assistance that is more based on valuing the individual, taking into account the user's social, economic and cultural context.

Keywords: Mental health; Mental health services; Unified Health System.

\section{Resumen}

La Reforma Psiquiátrica fue un proceso histórico cuyo principal objetivo fue asegurar la humanización de la atención a las personas que experimentan malestar psicológico. Este artículo busca en la literatura nacional, reflexionando sobre las vicisitudes de la dimensión relacional del cuidado, la constitución del RAPS como instrumento para promover la humanización en salud, la inclusión social y romper todo el estigma en torno a la salud mental. Este estudio tiene como objetivo reflexionar sobre la atención en salud mental que brinda la Red de Atención Psicosocial en Salud (RAPS). Se trata de un ensayo teórico que discute el cuidado de la salud mental desde la perspectiva de la Reforma Psiquiátrica, teniendo como referencia teórica el cuidado ontológico, visto bajo la fenomenología según Heidegger. La discusión sobre el tema se da en dos momentos, reflexionando sobre el rol del cuidado y la Reforma Psiquiátrica en la articulación de los servicios de salud mental. Por tanto, el cuidado implica una serie de principios teóricos y prácticos que son necesarios para ofrecer una asistencia más humana a la salud. Incluso frente a los retrocesos y debilidades que enfrenta la Salud Mental brasileña, los profesionales deben reflexionar y replantear la humanización de la atención en el contexto de la salud mental. Los profesionales de la salud mental deben buscar siempre una asistencia que se base más en la valoración del individuo, teniendo en cuenta el contexto social, económico y cultural del usuario.

Palabras clave: Salud mental; Servicios de salud mental; Sistema Único de Salud.

\section{Introdução}

O cuidado em Saúde Mental veio de constantes evoluções sociais que ocorreram ao longo dos tempos. Após períodos de grandes conflitos e ditadura foi aprovada a Constituição Federal de 1988 que trouxe muitas garantias aos cidadãos, especialmente no aspecto da saúde, juntamente com o direito à vida e à dignidade da pessoa humana. Saúde é considerada como direito social, dever de cuidado pelos três entes federados, União, Estados e Municípios, que devem fazê-lo da melhor maneira possível, impedindo que os cidadãos fiquem desamparados.

A Carta Magna traz uma sessão especial em seu texto que trata da saúde, dispondo através que o artigo 196 que a saúde é direito de todos e dever do Estado, garantido mediante políticas sociais e econômicas que visem à redução do risco de doença e de outros agravos e ao acesso universal e igualitário às ações e serviços para sua promoção, proteção e recuperação (Brasil, 1988).

Portanto, o Estado é precursor e responsável pela elaboração e implementação de políticas públicas. Consequentemente às disposições constitucionais é implementado o Sistema Único de Saúde (SUS), legitimado pela Lei ${ }^{\circ}$ 8.080, de 19 de setembro de 1990, com o objetivo de promoção, proteção, organização e recuperação da saúde. O SUS, uma inovação para a população brasileira, trouxe grande evolução na área da saúde, disponibilizou acessos àqueles que não conseguiam determinados tipos de tratamentos, sendo, portanto, uma grande conquista social (Brasil, 1990).

No âmbito da saúde mental, o SUS aumentou o acesso a tratamentos inovadores oferecidos numa rede de saúde direcionada à área. Com a aprovação da lei 10.216/2001 e o movimento da reforma psiquiátrica no Brasil, o modelo de atenção psicossocial à saúde foi implantado. Ele se caracteriza pela ampliação do conceito de saúde e incorpora o cuidado integral e humanizado, com vistas a superar a visão biomédica e a atenção individualizada (Brasil, 2001).

O modelo psicossocial proporciona olhar mais humanitário aos portadores de transtornos mentais e suas famílias, garante efetivamente os direitos e redireciona o modelo assistencial à saúde. Neste campo, o cuidado em Saúde Mental no Brasil é desenvolvido principalmente no contexto da Atenção Primária à Saúde (APS) e dos Centros de Atenção Psicossocial (CAPS), onde é planejado por meio do território e das necessidades do usuário. No que se refere à construção do cuidado, a literatura internacional destaca que as práticas territoriais de cuidado em saúde mental são dispositivos imprescindíveis na inserção e aceitação dos usuários na comunidade. A rede de atenção constituída pelos serviços de saúde mental deve propiciar 
uma assistência eficiente, resolutiva e de qualidade, que permita ao usuário a reinserção e o convívio social mediado pelas práticas territoriais. (Campos; Bezerra, \& Jorge, 2020; Correia, Barros, \& Colvero, 2011).

É notório e complexo todo o processo que envolve o cuidado em saúde mental (Cardoso \& Galera, 2011; Rotoli, Silva, Santos, Oliveira, \& Gomes, 2019), cabendo tratamentos polimedicamentosos, suporte terapêutico e ocupacional a longo prazo envolvendo diversos profissionais e serviços que visam a reabilitação e integração psicossocial. Diante disso, na prestação de assistência ao paciente portador de transtornos mentais é essencial a participação da equipe "multi, inter e transdisciplinares para a efetivação do SUS e dos valores da Reforma Psiquiátrica, bem como a atuação política e investimentos necessários na área" (Jafelice \& Marcolan, 2018, p. 2265).

Portanto, o paciente junto de sua família e os profissionais dos serviços comunitários são atores primordiais na oferta de cuidados, exigindo a articulação de diversos serviços ofertados pela rede de assistência em saúde. (Ayres, 2004). Apesar de sua importância, na prática destes serviços persistem adversidades complexas como a formação dos trabalhadores que atuam na área, a execução de ações intersetoriais, implementação de intervenções que valorizem as potencialidades do território como educação, esporte, trabalho, entre outros, a fim de compreender o "ser" indissociável no contexto que se vive com a garantia da sua participação no contrato social. (Campos, Bezerra, \& Jorge, 2020).

Considerando as potencialidades e obstáculos que perpassam os serviços de saúde mental, perguntamo-nos: Como se dá o cuidado no âmbito da rede de apoio psicossocial, no contexto da reforma psiquiátrica?

Diante dos retrocessos na Política Nacional de Saúde Mental desde 2016, os cortes de verbas da RAPS e o atual cenário político onde a Saúde Mental, corre sério risco de sofrer um desmonte, podendo perder seu modelo de atenção psicossocial, que foi conquistado a partir de grandes lutas e evidências científicas, é importante que venhamos refletir sobre as conquistas originadas através da Reforma Psiquiátrica e o papel do cuidado em Saúde Mental como forma de humanização. O presente estudo tem como objetivo realizar uma reflexão sobre o cuidado em saúde mental proporcionado pela Rede de Atenção Psicossocial à Saúde (RAPS).

\section{Metodologia}

Trata-se de um ensaio teórico que discute o cuidado em saúde mental na perspectiva da Reforma psiquiátrica dispondo do significado de cuidado descrito por de Heidegger (1995) em sua obra "Ser e Tempo" que empregou a ótica da fenomenologia. As reflexões sobre o cuidado em saúde mental na RAPS se deram a partir das interlocuções presentes na literatura acerca das potencialidades e obstáculos oportunizados pelo movimento de Reforma Psiquiátrica no âmbito do Sistema Único de Saúde. O ensaio é um meio de analisar determinado objeto independente da origem, traz novos conhecimentos científicos de modo leve e sem radicalismo. Não exige metodologia específica, pois trabalha de modo amplo, com a intenção de construir algo relevante para reflexão científica. A subjetividade está sempre presente e é elemento importante para ajudar no processo de conhecimento com originalidade (Meneguetti, 2011).

\section{Resultados e Discussão}

De acordo com a etimologia, "cuidar" é uma palavra de origem latina, frequentemente, ligada ao verbo "cogitare" cujo significado é atribuído à ação de pensar, supor e imaginar. Já o verbo "cogitare", origina-se do vocábulo "co-agitare", logo, assume o significado de "agitação de pensamento", "revólver no espírito" ou "tornar a pensar em alguma coisa". (Montenegro, 2001).

O cuidado é próprio da natureza humana, da nossa constituição enquanto seres racionais. Sob esta perspectiva é necessário buscar diferentes significados para uma compreensão do cuidado dentro de todas as dimensões que permeiam este 
conceito. Como também aliá-lo às condições físicas, mentais e sociais que envolve aquele que sofre com algum transtorno mental e a sua família que convive, apoia e é o maior suporte durante todo o processo terapêutico (Boff, 1999).

De acordo com Cardoso e Galera (2011) o cuidado em saúde mental vai ocorrer no estabelecimento de uma intrínseca relação entre os serviços de saúde, seus profissionais, o paciente e sua família, considerando as particularidades de cada contexto cultural, social e econômico. Para Viegas e Penna (2015) o conceito de integralidade está embasado em tratar a pessoa como ser humano, ou seja, com mais cuidado e delicadeza, atribuindo a ela o seu devido valor e respeito e acima de qualquer coisa, atender junto às suas necessidades, nessa condição o cuidado logo seria um cuidar do todo.

É na condição de um cuidar-se, um "tratar de ser", que é, de um lado, um ato individual e individualizado, mas, de outro lado, ou seja, é também uma relação necessária com o outro, com um mundo compartilhado que forja a identidade de todos e cada um, e que também se torna possível compreender a existência humana. (Heidegger, 1995).

O conceito de cuidado (Sorge) é definido como o ser do "ser-aî" (Dasein), ente principal da analítica existencial desenvolvida por Heidegger, através de sua obra Ser e Tempo, ele aborda a questão do Ser através do método fenomenológico, fazendo da reflexão acerca do Ser seu ponto de partida. Este autor aponta o fato de que, através do próprio homem, é que se dá o caminho para se conhecer o Ser. O homem em sua solidão interroga-se sobre si mesmo, colocando-se em questão e refletindo sobre ele mesmo, e neste momento o Ser dá-se a conhecer. O objetivo da reflexão filosófica encontra-se no fato de que o filósofo, partindo da existência humana (Dasein - ser-aí), procura desvendar o ser em si mesmo", ou seja, a preocupação maior de Heidegger não é o que é o ser humano, mas sim como é "ser" humano. (Nunes, 1992).

A obra Heideggeriana aborda vários conceitos muito importantes para a compreensão do cuidado ontológico. Como por exemplo o de: Cura: refere-se a uma das características ontológicas do ser-aí e diz respeito à condição do ser-aí cuidar, zelar, por suas possibilidades de "poder-ser". Do ponto de vista ôntico, todos os comportamentos e atitudes do homem são dotados de cura e guiados por uma "dedicação". Cuidado: cuidado pode ser entendido como ato, o qual ocupa um sentido ôntico, ou como possibilidade, um sentido que vai além do ato, além do que se pode perceber, ocupando um sentido ontológico. Para Heidegger o cuidado contempla o modo positivo de cuidar dos entes, não é sinônimo de bondade, é entender autenticamente o que é importante. Ôntico: tudo que é percebido, entendido, conhecido de imediato. É a compreensão cotidiana do ser em que nos movemos. É a dimensão do ser-aí envolvido na cotidianidade. Ontológico: dimensão ontológica diz respeito àquilo que antecede originariamente toda manifestação ôntica e lhe garante um sentido. Diz respeito às diferentes possibilidades de ação do ser-aí junto às coisas e aos outros, não no sentido daquilo que é manifesto, mas daquilo que possibilita toda a manifestação. (Oliveira \& Carraro, 2011).

O cuidado na visão de Martin Heidegger traz no sentido de "Cura" o ser de presença que aspirava conquistar os aspectos ontológicos adequados para o ente que nós mesmos somos e que damos o nome de "homem". Ontologicamente é compreendido por Heidegger como "cuidado" e "apreensão", ou seja, todas as ações comportamentais e de atitude do homem são dotadas de "cura" e guiados por uma "dedicação". No entanto a condição existencial de haver cuidado como cura e dedicação, deve ser concebido como cura sob uma visão autêntica, ou seja, como cuidado ontológico. (Viegas \& Penna, 2015). O "ser-no-mundo" já é sempre numa "de-cadência". Pode-se, portanto, determinar a cotidianidade mediana da "presença" como ser-no-mundo aberto na decadência que, lançado, se projeta e que, em seu ser junto ao mundo e em seu ser-com os outros, está em jogo o seu poder-ser mais próprio (Heidegger, 2005, p. 244).

O sentido de cuidado para Heidegger não está restrito apenas ao comportamento e a ideia de zelar pelas coisas e o bem-estar das pessoas, também nos traz um sentido dentro da ontologia de um ser autêntico que pode evitar "entificar" o ser que o levará a uma angústia existencial, para ele a angústia ocorre quando o ser se perde em si mesmo. (Heidegger, 2005).

Quando Heidegger aponta que o cuidado é fundamental para qualquer interpretação do ser humano e só é possível através do cuidado que o querer e o desejar emergem como realização do humano. Aqui compreendemos que não é possível 
pensar o cuidado como apenas teorização de ação, como também não se pode defini-lo como uma simples forma e única estrutura em si mesma, pois sua condição mostra uma articulação estrutural que se exprime de forma intrínseca (Viegas \& Penna, 2015). Pensando do cuidado centrado na essência do ser de Heidegger, e na maneira como a loucura fora conduzida, alienando, enclausurando, excluindo e estigmatizando todos aqueles que eram fora do padrão normal, sendo um tipo de incômodo para a sociedade, a reforma vem trazer com a assistência psicossocial não apenas a humanização do cuidado em saúde mental, mas ela também reconhece a singularidade, autonomia e os direitos das pessoas com transtornos psíquicos, devolvendo a identidade que essas pessoas ocupam na sociedade. Então nessa perspectiva a reforma vem reconhecer "como é ser o ser" que experimenta algum tipo de sofrimento psíquico.

As reflexões acerca da temática são desenvolvidas em dois momentos: 1. O cuidado; 2. Reforma Psiquiátrica: articulação de serviços rumo a um cuidado humanizado.

\subsection{O cuidado}

Segundo Boff (1999) cuidar seria a base do ser humano, desde seu nascimento até sua própria morte. Sem o cuidado, a pessoa definharia e perderia o sentido de sua existência e como consequência morreria. Cuidar seria a afirmação da capacidade do ser humano para com seu semelhante, presente até no gesto mais simples; "cuidar é mais que um ato, é uma atitude que representa ocupação, preocupação, responsabilização e envolvimento afetivo com o outro".

Cuidado como designação de uma atenção à saúde interessada no sentido existencial da experiência do adoecimento, físico ou mental, e, por conseguinte, também das práticas de promoção, proteção ou recuperação da saúde. (Castiel \& Povoa, 2001). O cuidado envolve uma série de princípios teóricos e práticos que são necessários para o oferecimento de uma assistência mais humana à saúde. (Ayres, 2004). Assim, o cuidado no âmbito da saúde mental deve ser pautado na valorização do indivíduo (Fidelis, 2018), levando em consideração o contexto social, econômico e cultural do cliente (Fidelis, 2018; Cardoso \& Galera, 2011). Entretanto, a oferta do cuidado em saúde mental não está limitada apenas em diminuir o risco de internação ou até mesmo controlar os sintomas. Na atualidade em que vivemos cuidar também compreende fatores pessoais, sociais, emocionais e recursos financeiros, fatores que são atribuídos ao próprio convívio com o processo de adoecimento mental. De modo que o cuidado passa a fazer parte do cotidiano e necessita de uma atenção que nem sempre é dispensada adequadamente devido a obstáculos presentes neste processo decorrentes de familiares, rede social, profissionais, sociedade e até mesmo do próprio paciente, como por exemplo a escassez de investimentos na área, assistência profissional ineficaz, estigma social, violação do direito dos pacientes, falta de acesso aos programas e entre outros (Furegato, 2009)

Portanto, o cuidado nessa área é considerado complexo e envolve uma equipe multiprofissional qualificada para responder efetivamente às necessidades de cuidado dos pacientes nos serviços de saúde (Marcolino, Fantinatti \& Gozzi, 2018). A realização da capacitação deve considerar não somente os profissionais de saúde, mas todos os indivíduos que fazem parte deste processo de cuidar como o profissional, o paciente, os seus familiares e a comunidade. Ao se melhorar a compreensão das pessoas em relação às doenças mentais, as barreiras que impedem a promoção de um cuidado digno são quebradas, bem como pode ser restaurado o potencial desses pacientes para a vida autônoma em comunidade (Cardoso \& Galera, 2011).

Assim, a capacitação e a educação em saúde se constituem em formas de enfrentamento de alguns obstáculos ao cuidado efetivo em saúde mental, alia-se a essa luta o cuidado individualizado. O conhecimento das características pessoais e do cotidiano desses pacientes podem fornecer informações importantes para se obter um planejamento assistencial de ações mais humanas, compartilhadas e coletivas essenciais para o oferecimento do cuidado em saúde mental. (Cardoso \& Galera, 2011).

No exercício do cuidar, seja individual ou coletivo, permeiam eventos de relações entre modos de ser no mundo, nas quais seres que cuidam e seres cuidados se entrelaçam numa dinâmica intersubjetiva recíproca e até imperceptível. Nesse ir e 
vir do cuidado, pessoas (entes que cuidam) emprestam percepções, emoções, sentimentos, valores e saberes ao fenômeno (o que aparece, pessoa que está sendo cuidada) para fazer ver a partir de si mesmo o que se é em si mesmo" (Silva, Francioni, Sena, Carraro, \& Radünz, 2005). Nesse sentido, o cuidado é entendido como ação que vai além de procedimentos técnicos, engloba envolvimento e compromisso com o outro, tornando-se, portanto, uma ação humanizada. É possível perceber que quando Heidegger aponta que o cuidado é fundamental para qualquer interpretação do ser humano e só é possível através do cuidado que o querer e o desejar emergem como realização do humano (Viegas \& pena, 2015).

Dentre os profissionais envolvidos no cuidado, está o enfermeiro. Seu trabalho no campo de saúde mental ao longo da história caracteriza-se como um processo de "transição de uma prática eminentemente hospitalar para tratamento dos "doentes mentais" para outra que incorpora novos princípios e conhecimentos, pautada na interdisciplinaridade e no reconhecimento do outro como ser humano, inserido em um contexto familiar e comunitário" (Waidman, Marcon, Pandini, Bessa, \& Paiano, 2012, p. 347). Nesta perspectiva, o enfermeiro além de acolher o indivíduo abordando sua vida no contexto político cultural e psicossocial, também deve oferecer ao paciente uma interação terapêutica, assumindo postura capaz de acolher, interagir e ouvir através de ações e instrumentos terapêuticos que promovam o reabilitar e, consequentemente visam a construção da melhoria na qualidade de vida dos sujeitos. (Villela \& Scatena, 2004).

$\mathrm{O}$ cuidado adequado propiciado por familiares, rede social, comunidade e profissionais, além do autocuidado do paciente, podem mudar a trajetória de pessoas que vivenciam cotidianamente as demandas do transtorno mental. Mas é preciso lembrar que o cuidado se dá primeiramente dentro de um serviço de saúde e mesmo que ocorra em outras áreas intersetoriais, comunitárias e domiciliares, é coordenado por profissionais de saúde presentes numa instituição. Nesse âmbito se faz mister refletirmos acerca do cuidado contextualizado pela Reforma Psiquiátrica.

\subsection{Reforma psiquiátrica: articulação de serviços rumo a um cuidado humanizado.}

A Reforma Psiquiátrica surge em prol de mudanças na assistência prestada aos indivíduos que eram estereotipados como loucos e submetidos a ações excludentes e de não cuidado. O movimento que aconteceu em âmbito internacional, teve como objetivo romper com as barreiras políticas e institucionais que davam foco ao modelo manicomial e asilar, por meio de conceitos que abrangiam o cuidado humanizado, centrado na reinserção social do indivíduo na comunidade. No Brasil, o processo de reintegração das pessoas em sofrimento psíquico se deu entre as décadas de 1970 e 1990, sendo concretizado somente em 2001 com a Lei 10.216, também denominada Lei Antimanicomial (Silva, Silva, Filho, Nery, \& Vilela, 2020).

Essa lei decorre de movimento político, social e jurídico, onde possibilitou a reestruturação e construção de novas políticas públicas no âmbito da saúde mental, que reconhecesse na prática, ações de cuidado efetivas e que melhorasse a qualidade de vida das pessoas em sofrimento mental. Tais ações foram perpassadas pelo movimento de desinstitucionalização, pela participação dos familiares na construção do projeto terapêutico e na garantia da proteção e dos direitos das pessoas portadoras de sofrimento psíquico. Desta forma, rompe-se com o modelo assistencial tradicional e instituiu-se um novo modelo de cuidado à saúde integrado e articulado com toda a rede de atenção em saúde (Silva et al., 2020).

A Reforma Psiquiátrica apresentou avanços consideráveis na reorganização do serviço de saúde mental no Brasil, visto que reverteu os gastos com leitos psiquiátricos de 80 mil em 1970 para 25.988 em 2014, com redução de 75,24\% dos gastos hospitalares e investindo na implantação da RAPS, em especial na expansão dos CAPS, às ações intersetoriais, as iniciativas de desinstitucionalização e fortalecimentos dos vínculos entre usuários e familiares (Sampaio \& Bispo Júnior, 2021; Amarante \& Nunes, 2018).

Importante enfatizar que mesmo com avanços relevantes na área de saúde mental após a reforma psiquiátrica, nos últimos anos têm sido observado diversos episódios que remetem para o desmonte de toda construção nesta área, posto que a Nota Técnica 11/2019 impôs mudanças na Política Nacional de Saúde Mental (PNSM) e nas ações sobre drogas, emergindo a 
inclusão do hospital psiquiátrico e comunidades terapêuticas na RAPS, sendo este último reprodução do modelo privatista e manicomial sob tratamentos terapêuticos baseados na abstinência.

Outro argumento que aponta à retrocedência, se dá aos investimentos financeiros aos ambulatórios "especializados", substituindo os equipamentos já existentes, como o da Atenção Primária à Saúde e RAPS, que desempenham um papel importantíssimo nos territórios e com alto potencial de resolutividade. E por fim, o incentivo ao uso da Eletroconvulsoterapia (ECT) como tratamento terapêutico, que foi foco de uso desenfreado e demasiado nos manicômios brasileiros (Silva et al., 2020).

Nesse sentido, embora a RAPS ainda detenha de barreiras de alcance e efetividade desejada, principalmente nos cuidados complexos em saúde mental, é fundamental questionamentos em defesa dos equipamentos existentes na RAPS em substituição às práticas que remetem ao paradigma manicomial e em prol de fortalecimento, ampliando os serviços e a inclusão de atendimentos à crise, a promoção subjetividade do sujeito, da potência da comunidade e em estratégias de geração de renda e desenvolvimento cultural. (Sampaio \& Bispo Júnior, 2021).

É notório observar também que no âmbito dos processos de cuidado ainda existe uma resistência de superar o modelo de cuidado biomédico (Barbosa, Martinhago, Hoepfner, Daré, \& Caponi, 2016). Tratar sobre saúde mental envolve diversos fatores evolutivos no decorrer da aquisição de direitos da população. A questão por muito tempo foi negligenciada, mas atualmente, após diversas lutas, algumas garantias foram dispostas e devem ser aplicadas. A reforma psiquiátrica é fruto de muito esforço num objetivo de proporcionar aos pacientes psiquiátricos tratamento adequado e a desinstitucionalização.

A partir da aprovação da Lei 10.216 de 2001, a internação passou a ser uma medida que poderia ser adotada em situação de exceção, ou seja, passou-se a considerar o paciente em transtornos mentais como pessoa de direitos. A Declaração Universal de Direitos Humanos de 1948 garante o tratamento digno a todos os seres humanos, logo deve-se abolir, para qualquer pessoa, tratamentos cruéis, degradantes, maus tratos e discriminações, o que deveria ser observado desde esta época. Recentemente, em 2019, foi aprovada a Resolução $\mathrm{n}^{\circ}$ 8, de 14 de agosto do Conselho Nacional dos Direitos Humanos, que dispõe sobre soluções preventivas de violação e garantidoras de direitos aos portadores de transtornos mentais e usuários problemáticos de álcool e outras drogas (Brasil, 2001; Brasil, 2019).

A Resolução também traz olhar mais cuidadoso em relação à saúde mental e a interligação com os usuários de drogas, para que haja a integralização social. Chama atenção para a necessidade de análise dos fatores que influenciam para a capacidade de execução do que é estabelecido em legislação, como situações econômicas, culturais, étnicas, familiares, geracionais, entre outros que influenciam na solução da questão. Para isso, é importante falar dos Centros de Atenção Psicossocial (CAPS), que é um serviço aberto e comunitário de saúde do Sistema Único de Saúde (SUS), é um modelo de atenção para saúde mental (Brasil, 2019, Ferreira et al., 2016).

O CAPS concretiza ações dispostas na lei da Reforma Psiquiátrica, o objetivo é de atenção diária àquelas pessoas que apresentam sofrimento psíquico de forma acolhedora, como também aos seus familiares. Busca reinserir na sociedade aquele paciente que estava em situação de internação hospitalar, abre portas para concretizar a recuperação, o centro faz uma ponte entre hospital e comunidade. Por se encontrarem em diversas localidades são considerados a porta de acesso da população, o mais próximo, que demonstra a importância da evolução legislativa neste aspecto, já que ainda muito progresso é necessário para melhoria dos atendimentos (Freitas, 2018).

De acordo com Pessoa, Jorge, Lourinho e Catrib (2018) fica evidente que a precarização do trabalho possui atributos importantes com a não efetivação da construção longitudinal do cuidado nos serviços de saúde, visto que os trabalhadores são submetidos ao regime de trabalho vulnerável, como é o caso da fragilidade dos vínculos contratuais que acarretam grande rotatividade dos profissionais e consequentemente afetam os projetos terapêuticos e a construção de vínculos. Importante destacar que a fragilidade afeta ainda na atuação política dos profissionais, visto que questionamentos ou enfrentamentos às 
situações de não-conformidade na prática tornam-se atos de punições e demissões por estarem em desacordo com gestores e coordenadores dos serviços. O estudo aponta ainda as más condições de trabalho, principalmente aquelas relacionadas à estrutura física e a indisponibilidade de materiais básicos para execução dos projetos propostos para cuidado aos pacientes, porém apontam, que mesmo em meio às dificuldades, a sensação de frustração, cansaço e constrangimento, conseguem desempenhar trabalho pautado na qualidade do cuidado efetivo e humanizado.

Outra importante ferramenta no campo da saúde mental está o apoio matricial, tido como uma estratégia integrativa entre a atenção psicossocial e a APS que visa superação de barreiras no planejamento do cuidado, onde propõe-se a superação do cuidado verticalizado para a construção do cuidado na interação das equipes, sobrepujando a lógica dos encaminhamentos, referência e contra referência. Esse dispositivo possibilita romper a atuação da Rede Atenção Psicossocial (RAPS) que ainda permanece centrada no modelo asilar e da invisibilidade, desenvolvendo suas ações intramuros do serviço, reduzindo o poder de ação das equipes nos territórios, que podem desenvolver ações que envolva o contexto sociocultural, familiar e político dos usuário, e também permitir reconhecer as singularidades e complexidades de cada usuário no seu território, marcado por suas histórias, relações de poder e sua forma de organização na sociedade. (Campos, Bezerra \& Jorge, 2020).

As experiências elucidadas expõem a verticalidade das relações do trabalho e a assimetria de poderes, onde o saber médico ocupa lugar de destaque, apontando para práticas biomédicas de assistência à saúde, ganhando relevância a doença e a cura e subestimando o olhar para o corpo, considerando a pessoa como sujeito histórico e de vida social. Tais circunstâncias corroboram para atuações isoladas, desacreditando na potência do cuidado a ser produzido pela equipe interdisciplinar (Pessoa, Jorge, Lourinho, \& Catrib, 2018).

Para Silva e Trajano (2012) a Política Nacional de Humanização e a Reforma Psiquiátrica originam os frutos de uma mesma discussão e encontram suas raízes pautadas na ética em defesa da vida. Neste ponto, encontra-se uma nova confluência para a presente transposição; o debate que deu origem à reforma psiquiátrica foi o mesmo que produziu os fundamentos para a política de humanização em nosso país. Assim, experiências de cuidado em saúde mental na lógica da clínica antimanicomial acabaram trazendo reflexão sobre a humanização do cuidado ao derrubar muros, buscar liberdade e a defesa dos direitos dos sujeitos com sofrimento psíquico.

Neste sentido, é preciso fortalecer o trabalho em equipe e interdisciplinar, possibilitando a construção da Saúde Mental pautada na reflexão do cuidado, tendo o diálogo como elemento central, onde proporcionará ao profissional o desenvolvimento da empatia e interesse efetivo pelo outro, estabelecendo escuta atenta e desarmada de estigma e preconceito, abandonando a arrogância ao falar para o outro e estabelecer o falar com o outro. A construção do cuidado desencadear-se-á como um excelente dispositivo de aproximação possibilitando a construção de práticas inovadoras e humanizadas no campo da Saúde Mental (Pessoa et al., 2018).

\section{Considerações Finais}

Este método possibilitou o conhecimento do cuidado em saúde mental no âmbito do modelo psicossocial implementado pelo movimento de Reforma Psiquiátrica e efetivado pela Lei Antimanicomial. O processo da RAPS buscou promover novos saberes e fazeres no campo da saúde mental, principalmente na mudança das relações sociais que se estabelecem com a loucura, com o cuidado humanizado e a reinserção social. Heidegger em sua obra "O ser e o tempo", apresenta dois principais discursos, regidos por lógicas diversas, que coexistem e fundamentam modos distintos de compreender o sujeito e o seu sofrimento e, consequentemente, modos diversos de cuidado, assim sendo o diálogo em saúde é centrado no sujeito e em seu saber sobre as próprias experiências de saúde e sofrimento.

Nessa perspectiva, somam-se avanços, mas também grandes desafios, como a superação do histórico do estigma da doença mental: fenômeno relacional demarcador de desigualdades, a qualificação dos profissionais, a alocação dos recursos 
para este setor e principalmente aos retrocessos recentes das normativas técnicas e políticas hegemônicas da saúde mental que colocam em risco o cuidado, a humanização e integralidade na assistência nos serviços de saúde mental.

Apesar dos benefícios que a Reforma Psiquiátrica e a luta Antimanicomial trouxeram, o número dos CAPS pelo país ainda é insuficiente para essa transformação paradigmática da humanização do cuidado. Infelizmente, ainda há tratamentos obsoletos e a reprodução de uma lógica instituída. Este estudo levou-nos a compreender as fragilidades e potencialidades da RAPS no processo de construção do cuidado, bem como possibilitará que os profissionais e gestores ressignifiquem suas práticas e saberes na mudança das condutas pessoais e coletivas a fim de que tais mudanças alcancem de forma positiva a humanização do cuidado na assistência das pessoas em sofrimento psíquico.

Assim, sugere-se que estudos futuros explorem o cuidado integral e humanizado com foco no indivíduo em condições de sofrimento psíquico, de modo que a assistência psicossocial seja garantida como atenção indispensável à existência humana, possibilitando novas reflexões quanto aos retrocessos apresentados em passado recente e da mesma maneira possibilitar-se-á compreender e inteirar-se da atuação dos Serviços de Saúde Mental frente às mudanças na Política Nacional de Saúde Mental, principalmente no que tange a humanização do cuidado e os avanços alcançados no cotidiano da atenção à Saúde Mental.

\section{Referências}

Amarante, P., \& Nunes, M. O. (2018). A reforma psiquiátrica no SUS e a luta por uma sociedade sem manicômios. Ciênc. saúde coletiva, $23(6), 2067-2074$. https://doi.org/10.1590/1413-81232018236.07082018

Ayres, J. R. C. M. (2004). O cuidado, os modos de ser (do) humano e as práticas de saúde. Saúde e soc, 13 (3, 16-29. https://doi.org/10.1590/S010412902004000300003

Bandeira, M., \& Barroso, S. M. (2005). Sobrecarga das famílias de pacientes psiquiátricos. J Bras Psiquiatr, 54(1), 34-46. https://www.scielo.br/j/rpc/a/NrX7MSyJDwLByHGBDxh5Dfw/?lang=pt\&format=pdf

Barbosa, V. F. B., Martinhago, F., Hoepfner, A. M. S., Daré, P. K., \& Caponi, S. N. C. (2016). O cuidado em saúde mental no Brasil: uma leitura a partir dos dispositivos de biopoder e biopolítica. Saúde debate, 40 (108). de https://doi.org/10.1590/0103-1104-20161080015

Brasil (1988). Constituição da República Federativa do Brasil. Centro Gráfico.

Brasil (2001). Lei n 10.216 de 06 de abril de 2001. Senado Federal. http://www.planalto.gov.br/ccivil_03/leis/leis_2001/110216.htm

Brasil (1990). Lei No 8.080 de 19 de setembro de 1990. Senado Federal. http://www.planalto.gov.br/ccivil_03/leis/leis_2001/110216.htm

Brasil (2019). Resolução No 8, de 14 de agosto de 2019. Diário Oficial da União. https://www.in.gov.br/web/dou/-/resolucao-n-8-de-14-de-agosto-de-20192121753

Boff, L. (1999). Saber cuidar: ética do humano, compaixão pela terra. Vozes;

Campos, D. B., Bezerra, I. C., \& Jorge, M. S. (2020). Produção do cuidado em saúde mental: práticas territoriais na rede psicossocial. Trab. educ. saúde, 18(1). https://doi.org/10.1590/1981-7746-sol00231

Castiel, L. D., \& Póvoa, E. C. (2001). Dr. Sackett \& "Mr. Sacketeer"... Encanto e desencanto no reino da expertise na medicina baseada em evidências. Cad. Saúde Pública, 17(1), 205-214. https://doi.org/10.1590/S0102-311X2001000100021

Cardoso, L., \& Galera, S. A. F. (2011). O cuidado em saúde mental na atualidade. Rev. esc. Enferm. 45 (3), 687-691. https://doi.org/10.1590/S008062342011000300020

Correia, V. R., Barros, S., \& Colvero, L. A. (2011) Saúde mental na atenção básica: prática da equipe de saúde da família. Rev Esc. Enferm, 45 (6), $1501-1506$. https://doi.org/10.1590/S0080-62342011000600032

Fidelis, A. C. Sentido do cuidado em saúde mental: sobre a rede de atenção psicossocial do Sistema Único de Saúde (2018). Trab. Educ. Saúde, 16 (2), 561582. http://dx.doi.org/10.1590/1981-7746-sol00126

Freitas, L. B. (2018). A evolução da saúde mental no brasil: reinserção social. Revista Científica Semana Acadêmica. 126(1). https://semanaacademica.org.br/system/files/artigos/a_evolucao_da_saude_mental_no_brasil_reinsercao_social_0.pdf

Ferreira, J. T., Mesquita, N. N. M., Silva, T. A., Silva, V. F., Lucas, W. J., \& Batista, E. C. (2016) Os Centros de Atenção Psicossocial (CAPS): Uma Instituição de Referência no Atendimento à Saúde Mental. Rev. Saberes, 4(1), 72-86. https://facsaopaulo.edu.br/wpcontent/uploads/sites/16/2018/05/ed5/7.pdf

Furegato, A.R.F. (2009). Mental health policies in Brazil [editorial]. Rev. Esc. Enferm, 43(2), 1-2. https://www.scielo.br/j/reeusp/a/NgMtWpbWD3hK3Kz3QFmHTHr/?lang=en 
Heidegger, Martin. (1995) Ser e Tempo. Tradução Márcia de Sá Cavalcanti. (2a ed.). Vozes.

Jafelice, G. T.; \& Marcolan, J. F. (2018) The multiprofessional work in the Psychosocial Care Centers of São Paulo State. Rev. Bras. Enferm. 71(5), 21312138. http://dx.doi.org/10.1590/0034-7167-2017-0300.

Marcolino, T. Q., Fantinatti, E. N., \& Gozzi, A. P. N. F. (2018) Comunidade de prática e cuidado em saúde mental: uma revisão sistemática. Trab. Educ. Saúde, 16(2), 643-658. http://dx.doi.org/10.1590/1981-7746-so100112

Meneguetti, F. K. (2011). O que é ensaio teórico? Rev. Adm. Contemp., 15(2). 320-332. https://www.scielo.br/j/rac/a/4mNCY5D6rmRDPWXtrQQMyGN/?format=pdf\&lang=pt

Montenegro, M.T. (2001) A educação moral como parte da formação para o cuidado na educação infantil. Tese de doutorado, Pontifícia Universidade Católica de São Paulo, SP, Brasil.

Nunes B. (1992) Passagem para o poético. Filosofia e poesia em Heidegger. (2a ed.). Ática.

Oliveira, M. F. V., \& Carraro, T. E. (2011) Cuidado em Heidegger: uma possibilidade ontológica para a enfermagem. Rev. Bras. Rnferm., 64(2), 376-380. http://www.scielo.br/scielo.php?script=sci_arttext\&pid=S0034-71672011000200025\&lng=en\&nrm=iso

Pessoa, K. L. V., Jorge, M. S. B., Lourinho, L. A., \& Catrib, A. M. F. (2018). Gestão do cuidado e interdisciplinaridade: desafios do cotidiano da atenção psicossocial. Rev. Salud Pública, 20(6), 692-698. https://www.scielosp.org/article/rsap/2018.v20n6/692-698/

Rotoli, A., Silva, M. R. S. D., Santos, A. M., Oliveira, A. M. N., \& Gomes, G. C. (2019). Mental health in Primary Care: challenges for the resoluteness of actions. Esc. Anna Nery, 23(2) https://doi.org/10.1590/2177-9465-ean-2018-0303

Sampaio, M. L., \& Bispo Júnior, J. P. (2021) Entre o enclausuramento e a desinstitucionalização: a trajetória da saúde mental no Brasil. Trabalho, Educação e Saúde, 19. https://www.scielo.br/pdf/tes/v19/1981-7746-tes-19-e00313145.pdf

Silva, T. A., Silva, A. S., Filho, I. E., Nery, A. A., \& Vilela, A. B. A. (2020) (Re) Visitando a reforma psiquiátrica brasileira: perspectivas num cenário de retrocessos. Av. Enferm. 38 (3), 380-386. http://www.scielo.org.co/scielo.php?script=sci_arttext\&pid=S0121-45002020000300380\&lng=en\&nrm=iso

Silva, L. W. S., Francioni, F. F., Sena, E. L. S., Carraro, T. E., \& Randünz, V. (2005) O cuidado na perspectiva de Leonardo Boff, uma personalidade a ser (re) descoberta na enfermagem. Rev Bras Enferm 58(4): 471-475. https://www.scielo.br/j/reben/a/LL8jXjvRjg8Gm5gyrHtGnNM/?lang=pt

Silva, R., \& Trajano, A. R. C. Humanização e reforma psiquiátrica: a radicalidade ética em defesa da vida. Polis e psique, 2(3), 16-36. https://seer.ufrgs.br/PolisePsique/article/view/40318

Viegas, S. M. F., \& Penna, C. M. M. (2015). As dimensões da integralidade no cuidado em saúde no cotidiano da Estratégia Saúde da Família no Vale do Jequitinhonha, MG, Brasil. Interface (Botucatu), 19(55), 1089-1100. https://doi.org/10.1590/1807-57622014.0275

Villela, S. C., \& Scatena, M. C. M. A. (2012) Enfermagem e o cuidar na área de saúde mental. Rev Bras Enferm, 57(6), 738-41. https://doi.org/10.1590/S003471672004000600022

Waidman, M. A. P., Marcon, S. S., Pandini, A., Bessa, J. B., \& Paiano, M. Nursing care for people with mental disorders, and their families, in Primary Care. Acta Paul. Enferm. 25(3), 346-351. http://dx.doi.org/10.1590/S0103-21002012000300005 